\title{
Characterization and Genomic Study of Phage vB_EcoS-B2 Infecting Multidrug-Resistant Escherichia coli
}

\author{
Yue Xu ${ }^{1,2 t}$, Xinyan Yu ${ }^{1,2 t}, Y u G u^{1,2}$, Xu Huang ${ }^{3,4}$, Genyan Liu ${ }^{3,4}$ and Xiaoqiu Liu ${ }^{1,2 *}$ \\ ${ }^{1}$ Key Laboratory of Pathogen Biology of Jiangsu Province, Nanjing Medical University, Nanjing, China, ${ }^{2}$ Department of \\ Microbiology, Nanjing Medical University, Nanjing, China, ${ }^{3}$ Department of Laboratory Medicine, The First Affiliated Hospital \\ with Nanjing Medical University, Nanjing, China, ${ }^{4}$ National Key Clinical Department of Laboratory Medicine, Nanjing, China
}

The potential of bacteriophage as an alternative antibacterial agent has been reconsidered for control of pathogenic bacteria due to the widespread occurrence of multi-drug resistance bacteria. More and more lytic phages have been isolated recently. In the present study, we isolated a lytic phage named vB_EcoS-B2 from waste water. VB_EcoS-B2 has an icosahedral symmetry head and a long tail without a contractile sheath, indicating that it belongs to the family Siphoviridae. The complete genome of vB_EcoS-B2 is composed of a circular double stranded DNA of 44,283 bp in length, with $54.77 \%$ GC content. vB_EcoS-B2 is homologous to 14 relative phages (such as Escherichia phage SSL-2009a, Escherichia phage JL1, and Shigella phage EP23), but most of these phages exhibit different gene arrangement. Our results serve to extend our understanding toward phage evolution of family Siphoviridae of coliphages. Sixty-five putative open reading frames were predicted in the complete genome of vB_EcoS-B2. Twenty-one of proteins encoded by vB_EcoS-B2 were determined in phage particles by Mass Spectrometry. Bacteriophage genome and proteome analysis confirmed the Iytic nature of vB_EcoS-B2, namely, the absence of toxin-coding genes, islands of pathogenicity, or genes through lysogeny or transduction. Furthermore, vB_EcoS-B2 significantly reduced the growth of E. coli MG1655 and also inhibited the growth of several multi-drug resistant clinical stains of E. coli. Phage vB_EcoS-B2 can kill some of the MRD E. coli entirely, strongly indicating us that it could be one of the components of phage cocktails to treat multi-drug resistant $E$. coli. This phage could be used to interrupt or reduce the spread of multi-drug resistant $E$. coli.

\footnotetext{
Keywords: bacteriophage vB_EcoS-B2, genome sequence, multi-drug resistance, comparative genome, mass spectrometry
}

\section{INTRODUCTION}

Escherichia coli is a gram-negative bacterium, which can cause intestinal (diarrhea) or extraintestinal (urinary tract infection, septicaemia, pneumonia, and meningitis) diseases in humans and animals (Cabal et al., 2016). Current antibiotic treatments used to treat E. coli often result in a spread of multidrug resistance (MDR) of E. coli (Lepape and Monnet, 2009). For example, E. coli strains produce Extended-Spectrum Beta Lactamase and metallo- $\beta$-lactamas, enzymes which make E. coli become resistant to several antibiotic drugs (Peña et al., 2006; Birgy et al., 2011). Currently, carbapenems appear to be the last 
available treatment for the severe infections caused by MDR E. coli. The recent finding of carbapenemase which hydrolyzes carbapenem may soon lead to cases with no therapeutic issue (Birgy et al., 2011). The emergence of carbapenemase and the lack of efficient antibiotics are alarming, therefore, the welltolerated, highly effective therapeutic alternatives are urgently needed (Birgy et al., 2011; Castanheira et al., 2017). This has encouraged researchers into returning to use bacteriophages (phages) as a supplement or substitute of antibiotics to treat infection caused by bacterial (Bolocan et al., 2016).

Although phages have been discovered over a century ago, phages continue to have a major impact on modern biological sciences, especially with the growth of interests in the microbiome (Haq et al., 2012; Laanto et al., 2017) and treating multidrug-resistant bacteria (Kutateladze and Adamia, 2010; Bolocan et al., 2016). Many reports described that various pathogenic E. coli have been killed by lysis phages (Bourdin et al., 2014). Therapeutic bacteriophages that efficiently lyse the E. coli O104:H4 outbreak strain could be selected easily from a phage bank or isolated from the environment (Merabishvili et al., 2012). Phage EC200PP was able to treat sepsis and meningitis infections induced by drug-resistant E. coli S242 (Pouillot et al., 2012). Fitzgerald-Hughes's study proved that Extended-Spectrum Beta Lactamase producing E. coli strains can be killed by commercially available and laboratory-isolated bacteriophages (FitzgeraldHughes et al., 2014). Dufour et al. determined that bacteriophage LM33-P1 can infect $\beta$-lactams and fluoroquinolones resistant E. coli strains efficiently both in vitro and in vivo (Dufour et al., 2016). The study on the efficient treatment of E. coliinduced pneumonia with two bacteriophages (536 P1 and 536 P7) showed that a combination of antibiotic treatment and phage therapy resulted in a $100 \%$ survival rate in VAP-infected mice (Dufour et al., 2015). Therefore, bacteriophages can be used as a supplement for antibiotics to treat the infection caused by bacteria.

Phages are the most abundant biological entities present on earth, providing an unlimited resource for possible phage applications (Srinivasiah et al., 2008). Phages flourish in oceans, soil, wastewater treatment plants, hot-water springs, and animal gut (Srinivasiah et al., 2008; Williamson et al., 2017). E. coli phages are commonly isolated from sewage, hospital waste water, polluted rivers, and fecal samples of humans or animals (Jamalludeen et al., 2009; Dalmasso et al., 2016; Snyder et al., 2016; Amarillas et al., 2017). Selection of wide spectrum phages or phages cocktail components for therapeutic preparation are key procedures to overcome the shortage of phage therapy, such as narrow host range of phages and emergence of bacterial resistance to phages (Regeimbal et al., 2016). Constructing the "right" cocktail is essential for achieving the maximum effectiveness of phage therapy (Bourdin et al., 2014; Schooley et al., 2017). It is also essential to have a set of well-characterized phages available for constructing the "right" cocktail to infect the broad range of bacterial pathogens (Sybesma et al., 2016). Therefore, isolating new phages and unraveling their genome sequence seems to be urgently needed to accumulate sufficient phage stocks for phage therapy.
In the present study, we isolated a lytic phage named vB_EcoSB2 from waste water. Transmission electron microscopy of vB_EcoS-B2 morphology revealed that it belongs to the Siphoviridae family. vB_EcoS-B2 significantly reduced the growth of E. coli under laboratory conditions. Prior to its application in phage therapy, we determined the genomic sequence and particle proteins of phage vB_EcoS-B2. Our results confirmed that $\mathrm{vB} \_$EcoS-B2 does not include virulent genes, islands of pathogenicity, or genes through lysogeny or transduction, which is a good candidate for phage therapy.

\section{MATERIALS AND METHODS}

\section{Bacterial Strains and Culture Conditions}

The bacterial strains used in this study were listed in Table 1. E. coli strains BL21 (DE3), DH5 $\alpha$, JM110, TOP10, BW25113, S17-1 were stocks in our lab. Thirty-five clinical isolates of E. coli were isolated from clinical samples of patients in the First Affiliated Hospital of Nanjing Medical University, Nanjing, China. All clinical strains which were used for phage host range determination have antibiotic resistance. Twenty-one of them are Extended-Spectrum Beta Lactamase producing stains. Five of them are metallo- $\beta$-lactamas (New Delhi metallo- $\beta$-lactamase-5) producing stains. All strains were grown in Luria-Bertani (LB) medium at $37^{\circ} \mathrm{C}$.

\section{Isolation and Propagation of Bacteriophages}

E. coli MG1655 was used for isolating and enriching virulent bacteriophage from waste water in Nanjing. Sewage sample was filtered using $0.45 \mu \mathrm{m}$ pore-size filters (Millipore, USA) to remove bacteria. Filtrates were added to E. coli MG1655 culture in early-log-phase at $37^{\circ} \mathrm{C}$ for $24 \mathrm{~h}$ with constant shaking to enrich the phages. Then the culture was centrifuged at $12,000 \mathrm{~g}$ for $10 \mathrm{~min}$ at $4^{\circ} \mathrm{C}$ to remove $E$. coli cells. The enriched phage suspension was tested for plaque formation with E. coli MG1655 using the double-layer agar plate method. Plaques formed on the plates after $12 \mathrm{~h}$ of incubation at $37^{\circ} \mathrm{C}$. Single plaque was picked to start the second (and subsequent) round of amplification. The infection cycle was repeated until the plaques were homogeneous. The phages were then amplified and stored at $4^{\circ} \mathrm{C}$.

\section{Purification of Phages}

Purification of vB_EcoS-B2 was carried out as described previously with slight modifications (Heo et al., 2009). Briefly, E. coli MG1655 culture at the early-log-phase $(\mathrm{OD} 600=0.4)$ was infected by phage vB_EcoS-B2 at $37^{\circ} \mathrm{C}$ for $3 \mathrm{~h}$ with shaking. Cell debris was removed by centrifugation $\left(14,000 \mathrm{~g}, 10 \mathrm{~min}, 4^{\circ} \mathrm{C}\right)$. The supernatant was passed through $0.45-\mu \mathrm{m}$-pore-size filters, yielding a crude extract of phage. Then the phage crude extract was concentrated by ultracentrifugation $\left(100,000 \mathrm{~g}, 2 \mathrm{~h}, 4^{\circ} \mathrm{C}\right)$, and the pellet containing phages was suspended in SM buffer $(5.8 \mathrm{~g} / \mathrm{L}$ $\mathrm{NaCl}, 2 \mathrm{~g} / \mathrm{L} \mathrm{MgSO}_{4} \cdot 7 \mathrm{H}_{2} \mathrm{O}, 50 \mathrm{ml} / \mathrm{L} 1 \mathrm{M}$ pH7.5 Tris- $\left.\mathrm{HCl}\right)$. The concentrated suspension was further purified by cesium chloride gradient centrifugation $\left(90,000 \mathrm{~g}, 20 \mathrm{~h}, 4^{\circ} \mathrm{C}\right)$. The phage zone was collected (about $1 \mathrm{~mL}$ ) and diluted into $10 \mathrm{ml} \mathrm{SM}$ buffer, followed by participation at $200,000 \mathrm{~g}$ for $3 \mathrm{~h}$ to remove $\mathrm{CsCl}$. Finally, the 


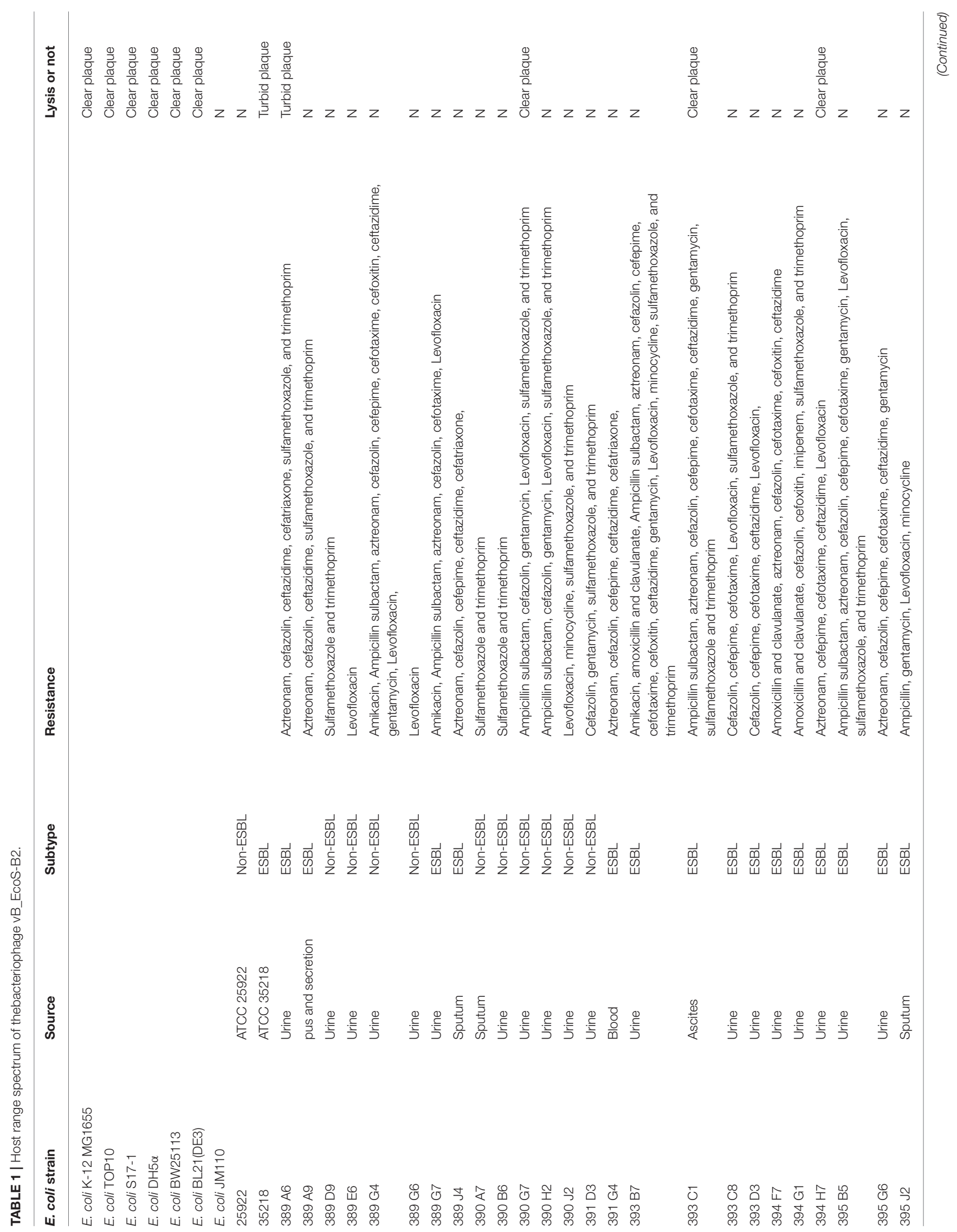




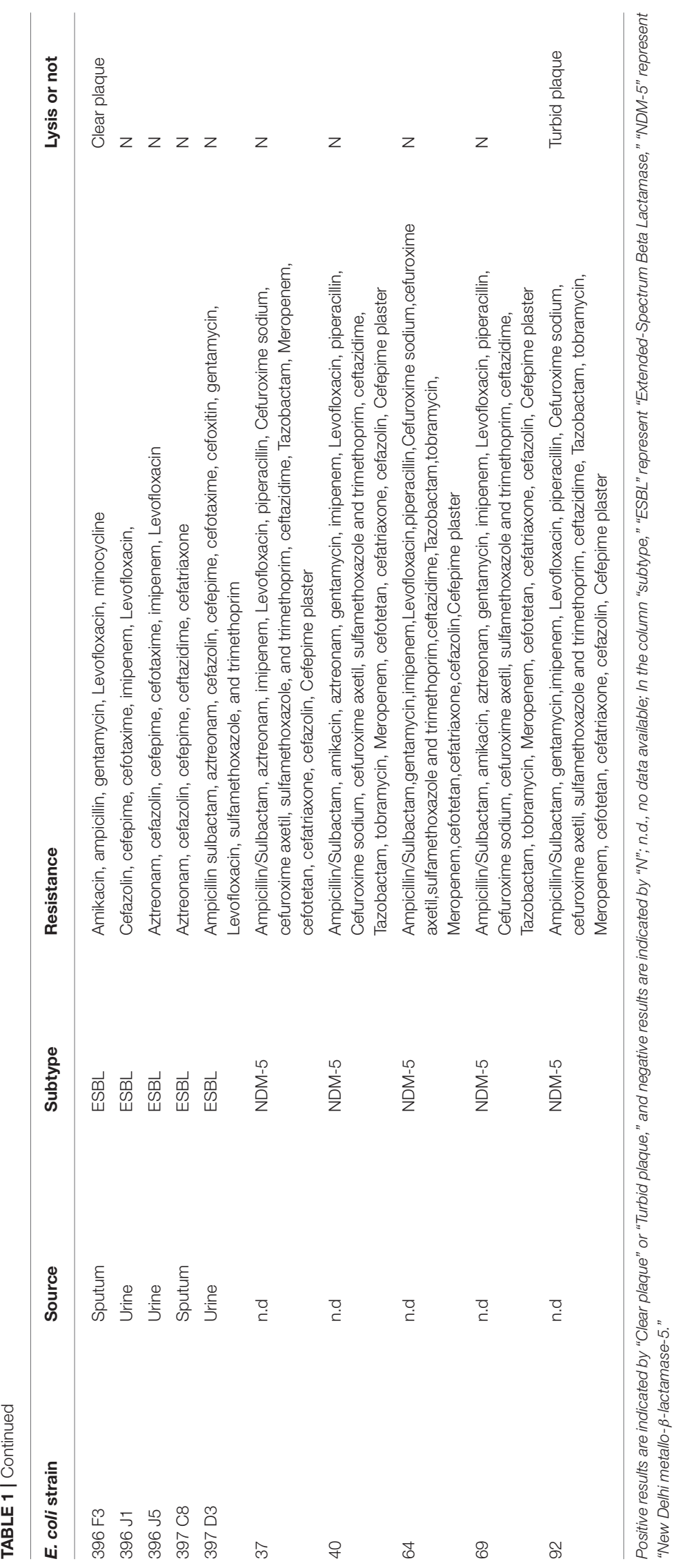


pellet was resuspended in SM buffer to yield the highly purified vB_EcoS-B2 particles.

\section{Electron Microscopy}

The purified phage particles of $\mathrm{vB}$ _EcoS-B2 were fixed on a copper grid with a carbon-coated film and negatively stained with $2 \%(\mathrm{w} / \mathrm{v})$ phosphotungstic acid. The micrographs were taken under FEI Tecnai G2 Spirit Bio TWIN transmission electron microscope at $80 \mathrm{kV}$.

\section{Temperature Stability}

The stability of bacteriophage under different temperature conditions was determined by constant temperature water bath method, a $1.5 \mathrm{~mL}$ tube containing $200 \mu \mathrm{L}$ (approximately $10^{10}$ $\mathrm{pfu} / \mathrm{mL}$ ) equal volumes of phage were incubated under different temperature $\left(4,25,37,45,50,55,60\right.$, and $\left.65^{\circ} \mathrm{C}\right)$. The phage titer was determined at intervals of $30 \mathrm{~min}$ from 0 to $180 \mathrm{~min}$, then at 6 and $24 \mathrm{~h}$ by the double-layer method. Three independent experiments were done and the value is represented by means.

\section{Host Range Analysis}

Ability of $\mathrm{vB} \_$EcoS-B2 to infect E. coli strains was tested. A total of $10^{9}$ cells were mixed with melted agar, and this mixture was poured on solid agar to make double-layer agar plates. After solidification, $10 \mu \mathrm{L}$ of phage suspensions (approximately $10^{10} \mathrm{pfu} / \mathrm{mL}$ ) of bacteriophage stock suspensions was spotted on plates carrying each bacterial strain. After adsorption of the spots, the plates were inverted and incubated for $24 \mathrm{~h}$ at $37^{\circ} \mathrm{C}$ before the degree of lysis was scored (Postic and Finland, 1961). All experiments were conducted according to the standard institutional guidelines of Nanjing Medical University (Nanjing, China). The study was approved by the research and ethics committee of the First Affiliated Hospital of Nanjing Medical University.

\section{One-Step Growth Curve}

One-step growth experiment was carried out as described previously with little modification (Yang et al., 2015). In brief, E. coli MG1655 was grown in LB medium until the early-logphase $\left(1 \times 10^{8} \mathrm{CFU} / \mathrm{mL}\right)$. Phage vB_EcoS-B2 was added to $E$. coli MG1655 culture at a multiplicity of infection (MOI) of 10 separately, and allowed to absorb for $10 \mathrm{~min}$ at $37^{\circ} \mathrm{C}$. Then the mixture was centrifuged at $14,000 \mathrm{~g}$ for $1 \mathrm{~min}$ to remove unadsorbed phages. After washing twice with fresh LB medium, the pellet of infected cells was resuspended in $50 \mathrm{~mL}$ of $\mathrm{LB}$ medium and the culture was continuously incubated at $37^{\circ} \mathrm{C}$. Using double-layer agar plate method, we determined the free bacteriophage count at each time point. The latency period and burst period were obtained directly from these one-step growth curves. The burst size of vB_EcoS-B2 was calculated by dividing the phage titers at plateau phase by the initial number of infective bacterial cells.

\section{Bacterial Challenge Assay}

For the bacterial challenge assay, $50 \mathrm{~mL}$ fresh LB broth was inoculated with an overnight culture of E. coli MG1655 ( $1 \%$ inoculum), followed by incubation at $37^{\circ} \mathrm{C}$ at 220 rpm until the OD600 was about 0.3. vB_EcoS-B2 stock solutions were then added $(\mathrm{MOI}=10)$ to these cultures. Bacterial growth was monitored by measuring the OD600 at various time points. As a negative control, bacterial cultures were inoculated with SM buffer instead of vB_EcoS-B2. OD600 was recorded at $15 \mathrm{~min}$ intervals, over a period of $300 \mathrm{~min}$.

\section{Extraction and Sequencing of the vB_EcoS-B2 Genome}

The purified phage sample was treated with DNase I (New England Biolabs) and RNase A (Tiangen Biotech) for $2 \mathrm{~h}$ at $37^{\circ} \mathrm{C}$ to digest the exogenous DNA and RNA. The preparation was then treated with proteinase $\mathrm{K}$ (Tiangen Biotech) for $15 \mathrm{~min}$ at $55^{\circ} \mathrm{C}$. The phage genome DNA was further prepared with a TIANamp Bacteria DNA Kit (Tiangen Biotech). DNA concentration was determined using a spectrophotometer (Nanodrop Technologies, USA). The vB_EcoS-B2 genomic DNA was sequenced using an Illumina HiSeq 2500 sequencer and reads were assembled into a whole genome using SOAPdenovov2.04 software and GapCloserv1.12.

\section{Annotation and Comparison}

Putative open reading frames (ORFs) were predicted using artemis software (http://www.sanger.ac.uk/science/tools/ artemis) and Glimmer 3 (Aggarwal and Ramaswamy, 2002), with a threshold of 30 amino acids (aa) as a minimum for the length of protein. Function annotation was performed using the BLAST tools at NCBI (http://blast.ncbi.nlm.nih.gov/Blast.cgi) against the non-redundant protein sequences database. Transfer RNAs (tRNAs) were identified using tRNAscan-SE (v1.23, http:// lowelab.ucsc.edu/tRNAscan-SE) and ribosome RNAs (rRNAs) were determined using RNAmmer (v1.2, http://www.cbs.dtu.dk/ services/RNAmmer/). DNAman was used to calculate molecular masses and isoelectric points for predicted phage proteins. The whole viral nucleotide sequence similarities between phages were determined by megablast analysis at NCBI. The global alignment of putative amino acid sequences was carried out by EMBOSS Needle tool at EMBL-EBI (European Molecular Biology Laboratory-European Bioinformatics Institute). Comparison of ORFs from relative phages was performed using EasyFig (http://mjsull.github.io/Easyfig/files.html) (Sullivan et al., 2011). Phylogenetic analyses between the genomes of related phages were performed with MEGA using the Neighbor-Joining algorithm.

\section{Structural Protein Analysis of vB_EcoS-B2}

The highly purified phage sample was subjected to sodium dodecyl sulfate polyacrylamide gel electrophoresis (SDSPAGE) using $12 \%$ acrylamide concentration. Gels were stained with silver as described by Shevchenko et al. (1996). For protein identification by liquid chromatography electrospray ionization with tandem mass spectrometry (LC-ESI MS/MS), the phage particles were digested with trypsin, and the tryptic peptides were analyzed by Q Exactive mass spectrometer (Thermo Scientific, USA). The corresponding ORFs were searched using MASCOT engine (Matrix Science, London, 
A

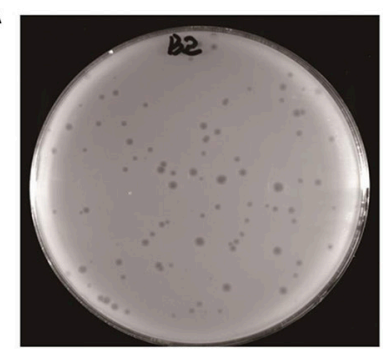

B

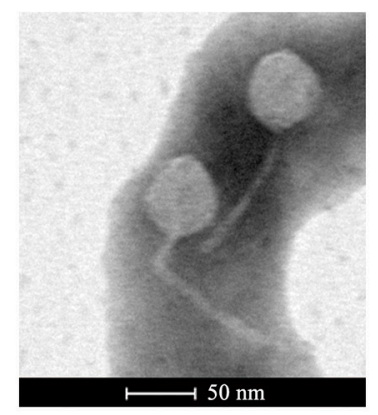

C

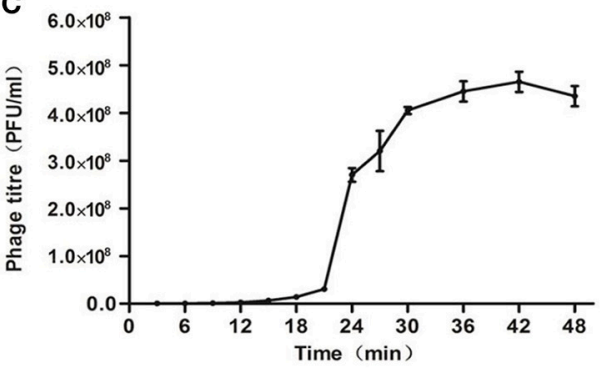

D

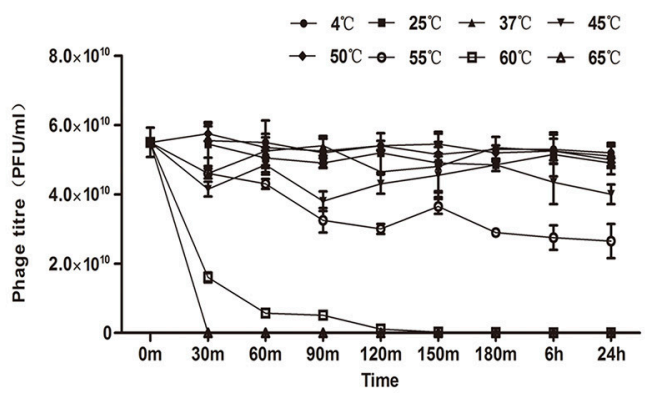

FIGURE 1 | Isolation, morphology, and biological properties of phage vB_EcoS-B2. (A) Plaque morphology of phage vB_EcoS-B2. (B) Transmission electron micrographs of E. coli phage vB_EcoS-B2. Bar indicates $50 \mathrm{~nm}$. (C) One-step growth curve of vB_EcoS-B2 on E. coli strain MG1655 at $37^{\circ} \mathrm{C}$. (D) Thermo stability of vB_EcoS-B2: the phages were incubated at different temperatures for $24 \mathrm{~h}$. Each value is the average from three different cultures \pm standard deviation in (C,D).

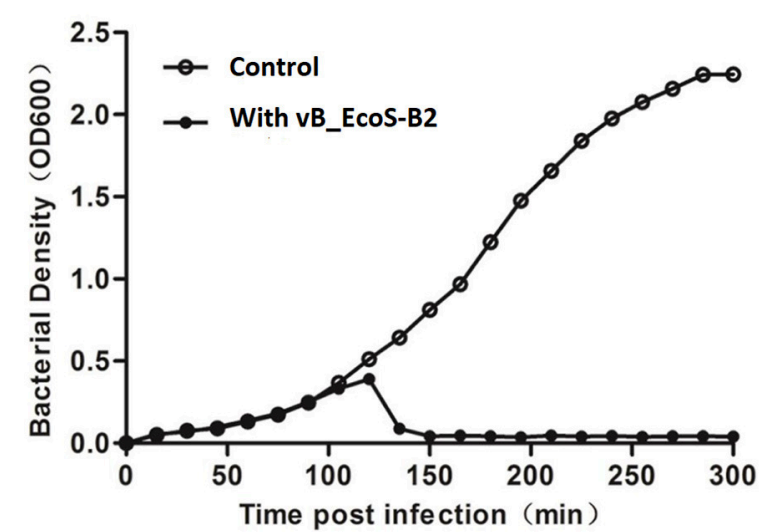

FIGURE 2 | Bacterial challenge assay with phage vB_EcoS-B2 to E. coli MG1655. vB_EcoS-B2 was added at an MOI of 10 to the bacterial culture after $1.5 \mathrm{~h}$ incubation (OD600 $=0.25$ ) (close circles). OD600 was recorded at $15 \mathrm{~min}$ intervals, over a period of $300 \mathrm{~min}$. A bacterial culture inoculated with SM buffer instead of vB_EcoS-B2 was used as the negative control (open circles). Each value is the average from three different cultures.

UK; version 2.2) against the protein sequence library of vB_EcoS-B2.

\section{Nucleotide Sequence Accession Number}

The genome sequence of vB_EcoS-B2 was deposited in GenBank under the accession number MG581355.

\section{RESULTS AND DISCUSSION}

\section{Phage Morphology}

A new E. coli phage vB_EcoS-B2 was isolated from wastewater in Nanjing. The phage formed clear round plaque (about 13 mm-diameter) with E. coli strain MG1655 after overnight culture at $37^{\circ} \mathrm{C}$ (Figure 1A). The purified phage particles of vB_EcoS-B2 were examined under the Transmission Electron Microscope. vB_EcoS-B2 has an icosahedral symmetry head and a long tail without a contractile sheath, indicating that it belongs to the family of Siphoviridae. The isometric head of vB_EcoSB2 had a mean diameter of $48 \mathrm{~nm}$, and the long non-contractile tail was about $143 \pm 6 \mathrm{~nm}$ (Figure 1B). vB_EcoS-B2 is closely resembled to the morphology of Enterobacteria phage SSL-2009a (an icosahedral head $62 \mathrm{~nm}$ in diameter and a long, flexible tail $138 \mathrm{~nm}$ in length) and Shigella phage EP23 (head diameter $59 \pm$ $3 \mathrm{~nm}$; non-contractile, filamentous tails $142 \pm 32 \mathrm{~nm}$ in length) (Li et al., 2010; Chang and Kim, 2011).

\section{Phage Population Dynamics}

One-step growth experiments were performed to assess the population kinetics of $\mathrm{vB} \_$EcoS-B2, in the presence of E. coli strain MG1655 (Figure 1C). vB_EcoS-B2 had latent period about $20 \mathrm{~min}$. All phages had been released by $30 \mathrm{~min}$ after infection (Figure 1C), and had a burst size of $224.1 \pm 10.7$ phage particles. Latent time of vB_EcoS-B2 was shorter than that of phage EP23 which had a latent period of $45 \mathrm{~min}$ (Chang and Kim, 2011). Latent and burst periods of $\mathrm{vB}$ _EcoS-B2 were very similar to that 
TABLE 2 | Predicted ORFs and genes encoded by the vB_EcoS-B2 genome.

\begin{tabular}{|c|c|c|c|c|c|c|c|}
\hline ORFs & Start & Stop & Directions & No. of residues & $M W(d a)$ & pl & Predicted molecular function \\
\hline ORF1 & 1 & 2,292 & + & 763 & 86167.7 & 8.56 & DNA polymerase I \\
\hline ORF2 & 2,292 & 2,570 & + & 92 & 10147.2 & 10.39 & Hypothetical protein \\
\hline ORF4 & 2,822 & 3,046 & + & 74 & 8761.5 & 9.94 & Hypothetical protein \\
\hline ORF5 & 3,051 & 4,475 & + & 474 & 53461.8 & 9.55 & DNA helicase \\
\hline ORF8 & 5,569 & 6,033 & + & 154 & 17882.3 & 8.19 & Hypothetical protein \\
\hline ORF9 & 6,020 & 6,505 & + & 161 & 18708.4 & 9.72 & DNA methyltransferase \\
\hline ORF10 & 6,486 & 6,710 & + & 74 & 8686.6 & 8.9 & Hypothetical protein \\
\hline ORF11 & 6,707 & 7,177 & + & 156 & 17872.8 & 6.25 & Hypothetical protein \\
\hline ORF12 & 7,242 & 7,796 & + & 184 & 20488.5 & 4.93 & Hypothetical protein \\
\hline ORF17 & 11,256 & 11558 & - & 100 & 10216.6 & 8.5 & Hypothetical protein \\
\hline ORF18 & 11,596 & 15,015 & - & 1,139 & 125892.9 & 5.11 & Putative tail fiber protein \\
\hline ORF19 & 15,012 & 15629 & - & 205 & 21245.4 & 8.71 & Putative tail assembly protein I \\
\hline ORF20 & 15,620 & 16,360 & - & 246 & 27577.5 & 4.95 & Tail assembly protein \\
\hline ORF21 & 16,363 & 17,151 & - & 262 & 28806.3 & 4.88 & Putative minor tail protein $\mathrm{L}$ \\
\hline ORF22 & 17,148 & 17,747 & - & 199 & 21696.3 & 6.52 & Putative minor tail protein \\
\hline ORF23 & 17,784 & 20,426 & - & 880 & 93307.2 & 9.93 & Putative tail length tape-measure protein 1 \\
\hline ORF24 & 20,426 & 20,518 & - & 30 & 3341.4 & 10.5 & Hypothetical protein \\
\hline ORF25 & 20,490 & 20,681 & - & 63 & 6931.5 & 8.51 & Hypothetical protein \\
\hline ORF26 & 20,821 & 21,051 & - & 76 & 8543.3 & 5.77 & Hypothetical protein \\
\hline ORF34 & 24,388 & 25,488 & - & 366 & 38395.4 & 7.66 & Major head protein \\
\hline ORF35 & 25,586 & 26,287 & - & 233 & 25384.1 & 5.51 & Hypothetical protein \\
\hline ORF36 & 26,388 & 26,756 & + & 122 & 13128.6 & 5.65 & Hypothetical protein \\
\hline ORF37 & 26,796 & 27,560 & + & 254 & 28937.1 & 8.29 & Hypothetical protein \\
\hline ORF38 & 27,574 & 27,732 & - & 52 & 5739.7 & 4.33 & Hypothetical protein \\
\hline ORF39 & 27,686 & 28,015 & - & 109 & 11931.4 & 9.88 & Hypothetical protein \\
\hline ORF40 & 28,008 & 29,111 & - & 367 & 40988.6 & 9.94 & Head morphogenesis protein \\
\hline ORF41 & 29,095 & 30,615 & - & 506 & 55192.3 & 4.51 & Phage structural protein \\
\hline ORF42 & 30,627 & 32,012 & - & 461 & 52196.7 & 7.52 & Terminase large subunit \\
\hline ORF43 & 32,012 & 32,584 & - & 190 & 21084 & 8.05 & Putative terminase small subunit \\
\hline ORF44 & 32,694 & 33,836 & - & 380 & 42737.4 & 7.35 & Putative phosphoesterase \\
\hline ORF45 & 33,857 & 34,057 & + & 66 & 7221.1 & 8.22 & Hypothetical protein \\
\hline ORF46 & 34,074 & 34,241 & - & 55 & 5603.2 & 4.48 & Hypothetical protein \\
\hline ORF47 & 34,258 & 34,749 & - & 163 & 18156.3 & 9.41 & Lysozyme \\
\hline ORF48 & 34,736 & 34,981 & - & 81 & 8870 & 8.51 & Putative holin-like class I protein \\
\hline ORF49 & 34,978 & 35,268 & - & 96 & 10183.1 & 8.23 & Putative holin-like class II protein \\
\hline ORF50 & 35,323 & 35,622 & - & 99 & 11796 & 9.72 & Hypothetical protein \\
\hline
\end{tabular}


TABLE 2 | Continued

\begin{tabular}{|c|c|c|c|c|c|c|c|}
\hline ORFs & Start & Stop & Directions & No. of residues & MW(da) & pl & Predicted molecular function \\
\hline ORF51 & 35,687 & 35,833 & - & 48 & 5626.5 & 11.71 & Hypothetical protein \\
\hline ORF52 & 35,830 & 36,363 & - & 177 & 20721.2 & 10.59 & Hypothetical protein \\
\hline ORF53 & 36,360 & 36,494 & - & 44 & 5107.7 & 3.43 & Hypothetical protein \\
\hline ORF54 & 36,485 & 36,670 & - & 61 & 7413.4 & 11.48 & Hypothetical protein \\
\hline ORF55 & 36,770 & 37,075 & - & 101 & 11091.8 & 10.31 & Hypothetical protein \\
\hline ORF56 & 37,072 & 37,206 & - & 44 & 5206.2 & 8.24 & Hypothetical protein \\
\hline ORF57 & 37,297 & 37,554 & - & 85 & 9233.9 & 11.49 & Hypothetical protein \\
\hline ORF58 & 37,609 & 39,861 & - & 750 & 82443.1 & 6.15 & Hypothetical protein \\
\hline ORF59 & 39,872 & 40,201 & - & 109 & 12545.1 & 8.47 & DNA recombination nuclease inhibitor gamma \\
\hline ORF60 & 40,424 & 41,032 & + & 202 & 23053.6 & 4.52 & Hypothetical protein \\
\hline ORF61 & 41,081 & 41,332 & + & 83 & 9953.6 & 9.91 & Hypothetical protein \\
\hline ORF62 & 41,333 & 41,521 & + & 62 & 7168 & 8.22 & Hypothetical protein \\
\hline ORF63 & 41,521 & 42,951 & + & 476 & 52319.8 & 8.73 & Hypothetical protein \\
\hline ORF64 & 42,944 & 43,186 & + & 80 & 9323.4 & 6.49 & Hypothetical protein \\
\hline ORF65 & 43,277 & 44,059 & + & 260 & 29909.5 & 4.55 & Hypothetical protein \\
\hline
\end{tabular}

of phage SSL-2009a which have latent and burst periods 10-15 and 30-40 $\mathrm{min}$ ( $\mathrm{Li}$ et al., 2010), respectively. Even though the burst size of vB_EcoS-B2 is less than that of SSL-2009a (about 375 PFU per infected cell) (Li et al., 2010), it is still a large burst size. As an antibacterial agent, a phage with a large burst size may indicate an easy selective advantage since phages with large burst sizes can increase the initial dose of phages several hundredfolds in short periods of time (Nilsson, 2014). The phages to be used in phage therapy must be strictly virulent and should be reproduced effectively and rapidly (Nilsson, 2014). Therefore, the large burst size and short latent time of vB_EcoS-B2 make it a good candidate for being used as the biocontrol agent against bacterial pathogens.

\section{Thermal Stability}

The thermal stability test was determined at different temperatures $\left(4,25,37,45,50,55,60\right.$, and $\left.65^{\circ} \mathrm{C}\right)$ whithin $24 \mathrm{~h}$ (Figure 1D). Results showed that the biological activity of phage vB_EcoS-B2 did not show any difference within the temperature ranging from 4 to $50^{\circ} \mathrm{C}$, but decreased sharply when the temperature increasing above $55^{\circ} \mathrm{C}$. Even though vB_EcoS-B2 does not like SSL-2009a which keeps activity more than 45 min over $63^{\circ} \mathrm{C}$ (Li et al., 2010), vB_EcoS-B2 is stable at relatively low temperature for longer time (Supplementary Figures 1,2). The phage is stable over a range of temperatures $\left(4-50^{\circ} \mathrm{C}\right)$ for $24 \mathrm{~h}$, suggesting that it has a good thermal stability and therefore easy to be preserved.

\section{Host Range and Effect of Phages on E. coli Growing Culture}

The ability of newly isolated phage to lyse E. coli strains was assayed by the spot test. vB_EcoS-B2 can infect some of the wellknown E. coli strains and several clinical MRD E. coli stains (Table 1). vB_EcoS-B2 is similar to SSL-2009a that is able to infect some engineered E. coli strains (Table 1). vB_EcoS-B2 can also infect the E. coli strain ATCC 35218, which could not be infected by SSL-2009a (Li et al., 2010). JL1 is a phage which can infect E. coli O157:H7 (Pan et al., 2013). EP23 can infect the three E. coli strains and two S. sonnei strains (Chang and Kim, 2011). The observations suggested that these very similar phages have different host range. Phage vB_EcoS-B2 only targeted seven MDR E. coli strains out of the 35 clinical isolates (Table 1). Therefore, Phage vB_EcoS-B2 has a relative narrow host spectrum. To overcome the narrow host range of phages, it is generally accepted that cocktails of multiple phages can be used to kill the great diversity of E. coli strains (Schmerer et al., 2014).

The effect of $\mathrm{vB}$ _EcoS-B2 alone on the growth of strain MG1655 was tested at MOI = 10 after $300 \mathrm{~min}$ of the phage added into the strain. vB_EcoS-B2 can efficiently reduce the growth of E. coli MG1655 after $30 \mathrm{~min}$ of infection (Figure 2, Supplementary Figure 3). Even though its host-range is relatively narrow, vB_EcoS-B2 has the possibility to be used as biocontrol agents against $E$. coli since it had strong lytic ability to $E$. coli. Therefore, it is essential to analyze complete sequence of vB_EcoS-B2 to ensure that the genome of vB_EcoS-B2 did not encode any genes associated with toxins, virulence factors, or lysogenic proteins.

\section{Basic Characteristics of vB_EcoS-B2 Genome}

The complete genome of $\mathrm{vB} \_$EcoS-B2 is composed of a circular double stranded DNA of 44,283 bp in length, with $54.77 \%$ GC content which was slightly higher than those of E. coli (50.6\%). Sixty-five putative open reading frames (ORFs) were predicted in the complete genome of vB_EcoSB2. The annotation of the properties of phage vB_EcoS-B2 genome, such as positions, directions, and putative functions of each gene were summarized in Table 2. Twenty-three ORFs were in the direct strand of the phage genome and 42 of them were in complementary strand (Figure 3). Only 25 ORFs (38.5\%) were predicted and determined to be putative functional (different colors), whereas 40 ORFs were assigned to hypothetical proteins (black color) (Figure 3). The 25 predicted functional proteins were categorized into five functional groups: DNA 


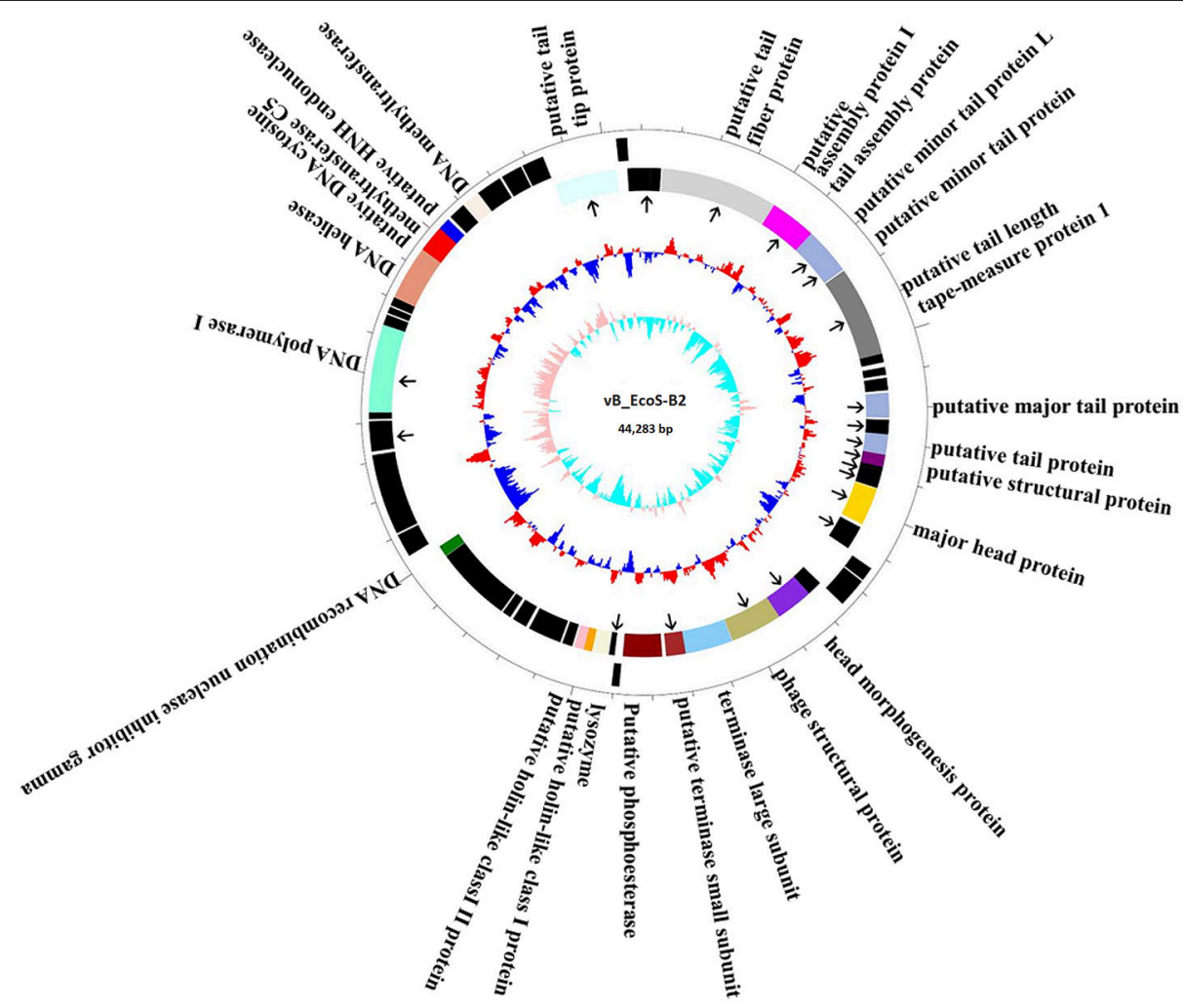

FIGURE 3 | Map of the genome organization of bacteriophage vB_EcoS-B2. The predicted ORFs are indicated as different colors in first circle. The outward showed the forward transcription genes and the inward showed the reversed transcription genes. Different colors identify ORFs with predicted molecular function. Hypothetical proteins marked by black. The second circle shows the $\mathrm{G} / \mathrm{C}$ content. Red outward indicated that the $\mathrm{G} / \mathrm{C}$ content of this region is higher than the average $\mathrm{G} / \mathrm{C}$ content of the whole genome, and blue inward indicated G/C content of this region less than the average. The third circle shows the GC skew. Particle proteins identified by Mass spectrometry were pointed by black arrows.

replication/modification (DNA polymerase I, DNA helicase, putative DNA cytosine methyltransferase $\mathrm{C} 5$, putative $\mathrm{HNH}$ endonuclease, DNA methyltransferase, DNA recombination nuclease inhibitor gamma), host lysis (lysozyme, putative holinlike class I protein, and putative holin-like class II protein), packaging (terminase large subunit, putative terminase small subunit), structural proteins (putative tail tip protein, putative tail fiber protein, putative tail assembly protein I, tail assembly protein, putative minor tail protein $\mathrm{L}$, putative minor tail protein, putative tail length tape-measure protein 1, putative major tail protein, putative tail protein, putative structural protein, major head protein, head morphogenesis protein, and phage structural protein), and additional functions (putative phosphoesterase) (Table 2, Figure 3). In addition, in vB_EcoS-B2 genome no statistically significant BLASTP similarity was identified in genes encoding integrase, recombinase, repressor, and excisionase (markers of temperate bacteriophages) (Carrias et al., 2011). Consequently, the vB_EcoS-B2 phage should be considered as a virulent bacteriophage. Genome analysis also suggests that the phage vB_EcoS-B2 does not encode genes associated with toxins or other virulence factors. Virulent characteristics and no possible pathogen factors make it feasible to be a potential candidate for therapeutic application.

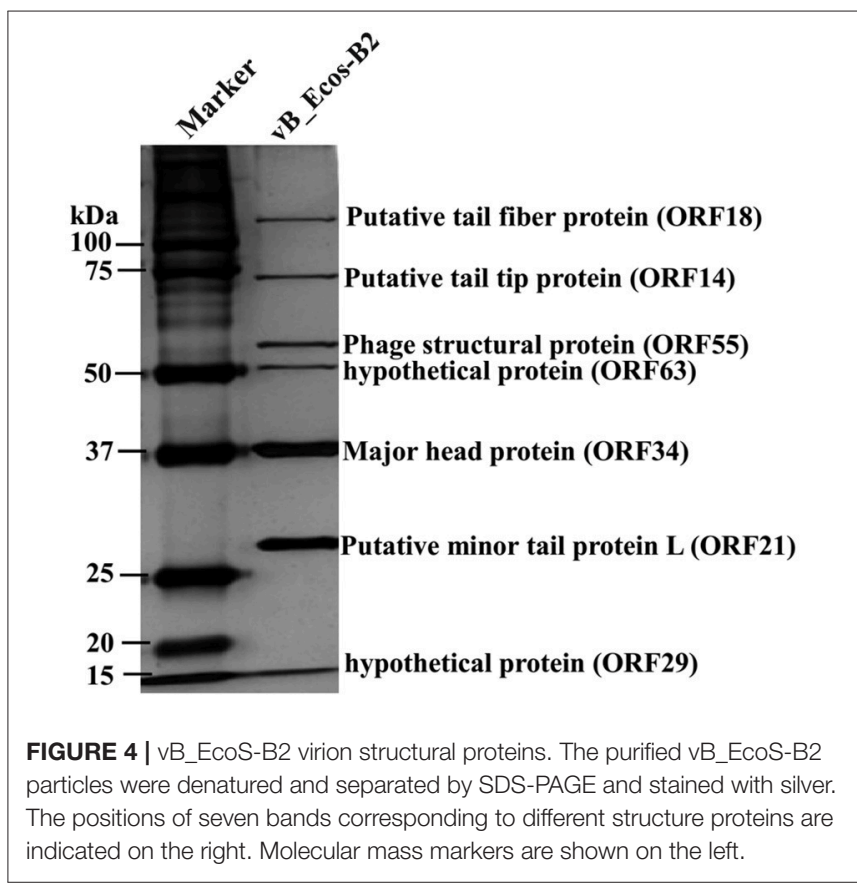


TABLE 3 | Mass spectrometry data for vB_EcoS-B2.

\begin{tabular}{|c|c|c|c|c|c|}
\hline ORFs & Predicted molecular function & Peptides & Unique peptides & Unique sequence coverage [\%] & $M W(d a)$ \\
\hline ORF23 & Putative tail length tape-measure protein 1 & 70 & 70 & 77.4 & 93307.2 \\
\hline ORF18 & Putative tail fiber protein & 52 & 52 & 59.2 & 125892.9 \\
\hline ORF34 & Major head protein & 40 & 40 & 86.1 & 38395.4 \\
\hline ORF14 & Putative tail tip protein & 36 & 36 & 56.7 & 65194.4 \\
\hline ORF41 & Phage structural protein & 31 & 31 & 72.7 & 55192.3 \\
\hline ORF28 & Putative major tail protein & 25 & 25 & 83.9 & 25755.3 \\
\hline ORF40 & Head morphogenesis protein & 24 & 24 & 61 & 40988.6 \\
\hline ORF21 & Putative minor tail protein $L$ & 18 & 18 & 63.4 & 28806.3 \\
\hline ORF44 & Putative phosphoesterase & 13 & 13 & 36.6 & 42737.4 \\
\hline ORF35 & Hypothetical protein & 13 & 13 & 56.2 & 25384.1 \\
\hline ORF22 & Putative minor tail protein & 11 & 11 & 51.5 & 21696.3 \\
\hline ORF30 & Putative tail protein & 9 & 9 & 43.1 & 21918 \\
\hline ORF33 & Hypothetical protein & 7 & 7 & 54.2 & 18924.7 \\
\hline ORF31 & Putative structural protein & 7 & 7 & 58.7 & 13053.8 \\
\hline ORF46 & Hypothetical protein & 5 & 5 & 94.5 & 5603.2 \\
\hline ORF16 & Hypothetical protein & 5 & 5 & 38.6 & 23211.6 \\
\hline ORF63 & Hypothetical protein & 4 & 4 & 15.4 & 52319.8 \\
\hline ORF29 & Hypothetical protein & 3 & 3 & 41.1 & 15087.1 \\
\hline ORF1 & DNA polymerase I & 3 & 3 & 8.5 & 86167.7 \\
\hline ORF32 & Hypothetical protein & 2 & 2 & 22.3 & 3990.7 \\
\hline ORF19 & Putative tail assembly protein I & 2 & 2 & 21 & 21245.4 \\
\hline
\end{tabular}

Particles proteins detected by MS are listed with their predicted functions and molecular mass. Molecular mass was calculated from the amino acid sequence of each gene. Number of identified peptides and unique peptides in each protein and the corresponding unique peptide sequence coverage are also indicated.

\section{Structural Proteins of vB EcoS-B2}

In order to analyze the structural proteins of $\mathrm{vB} \_$EcoS-B2, purified phage particles were denatured by boiling with sample buffer, and then separated by SDS-PAGE. At least seven distinct protein bands, with molecular weights ranging from 28.8 to 125.9 $\mathrm{kDa}$, were visualized in the SDS-PAGE gel (Figure 4). Five bands were identified as phage structural proteins (Major head protein, Putative minor tail protein L, Phage structural protein, Putative tail tip protein, and Putative tail fiber protein), and two bands were identified as hypothetical proteins (Figure 4). To determine every structural protein, phage particle proteins were identified by mass spectrometry, and results were listed in Table 3 . Twenty-one proteins were identified in mass spectrometry, including proteins corresponding to all seven distinct bands on SDS-PAGE gel (Table 3, Figure 4). Twelve out of 13 known structural proteins were identified by mass spectrometry, and some hypothetical proteins (ORF16, ORF29, ORF32, ORF33, ORF35, ORF46, ORF63) were also identified, which may be some unknown structural proteins. Interestingly, DNA polymerase I and putative phosphoesterase were determined in our phage particles. DNA polymerase I could fill DNA gaps during DNA repair, recombination, and replication (Andraos et al., 2004). DNA polymerase I and putative phosphoesterase may play important roles in the phage early infection processes, which will be our interests in our further studies.

\section{Comparative Genome Analysis}

Based on the result of BLAST analyses, the genome sequence of vB_EcoS-B2 displays significant similarity (coverage 87-95\%, identity $90-94 \%)$ to 14 phages isolated from different regions around the world, suggesting that the complex evolutionary relationships exist among these phages. The corresponding genome sequences of these phages were aligned, concatenated, and a phylogenetic tree was built using the maximum likelihood method (Figure 5). Phylogenetic tree of these phages has two main branches. Phylogenetic tree analysis showed that vB_EcoS-B2 is a novel bacteriophage that is closely related to phages JL1, YD-2008.s, Sloth, Envy, slur05, and lust, and relatively distant to phages SSL-2009a, HK578, EK99P-1, Sodalis phage SO-1, Shigella phage EP23, slur06, Gluttony, and Pride (Figure 5). The results of the comparative genomic analyses extend our understanding of the evolution and relationship between vB_EcoS-B2 and its bacteriophage relatives.

Most of the proteins from vB_EcoS-B2 and 14 relative phages are homology with each other. But some blank regions could be observed though genome comparison of these phages (Figure 6). The phage tail tip protein encoded by ORF14 shows a greater divergence between these phages (43-99\% coverage and $68-85 \%$ identity) (Figure 6). The ORF14 is relatively similar to tail fiber protein of phage Envy (99\% coverage and 70\% identity). The tail proteins are thought to be involved in host recognition, and confer the phage host range specificity (Hashemolhosseini et al., 1994). The small differences in tail fiber proteins are often associated with significant differences in host ranges and other biological properties (Yosef et al., 2017). This putative tail tip protein of $\mathrm{vB}_{-}$EcoS-B2 is relatively different from tail tip/fiber proteins of other phages, which may make the 

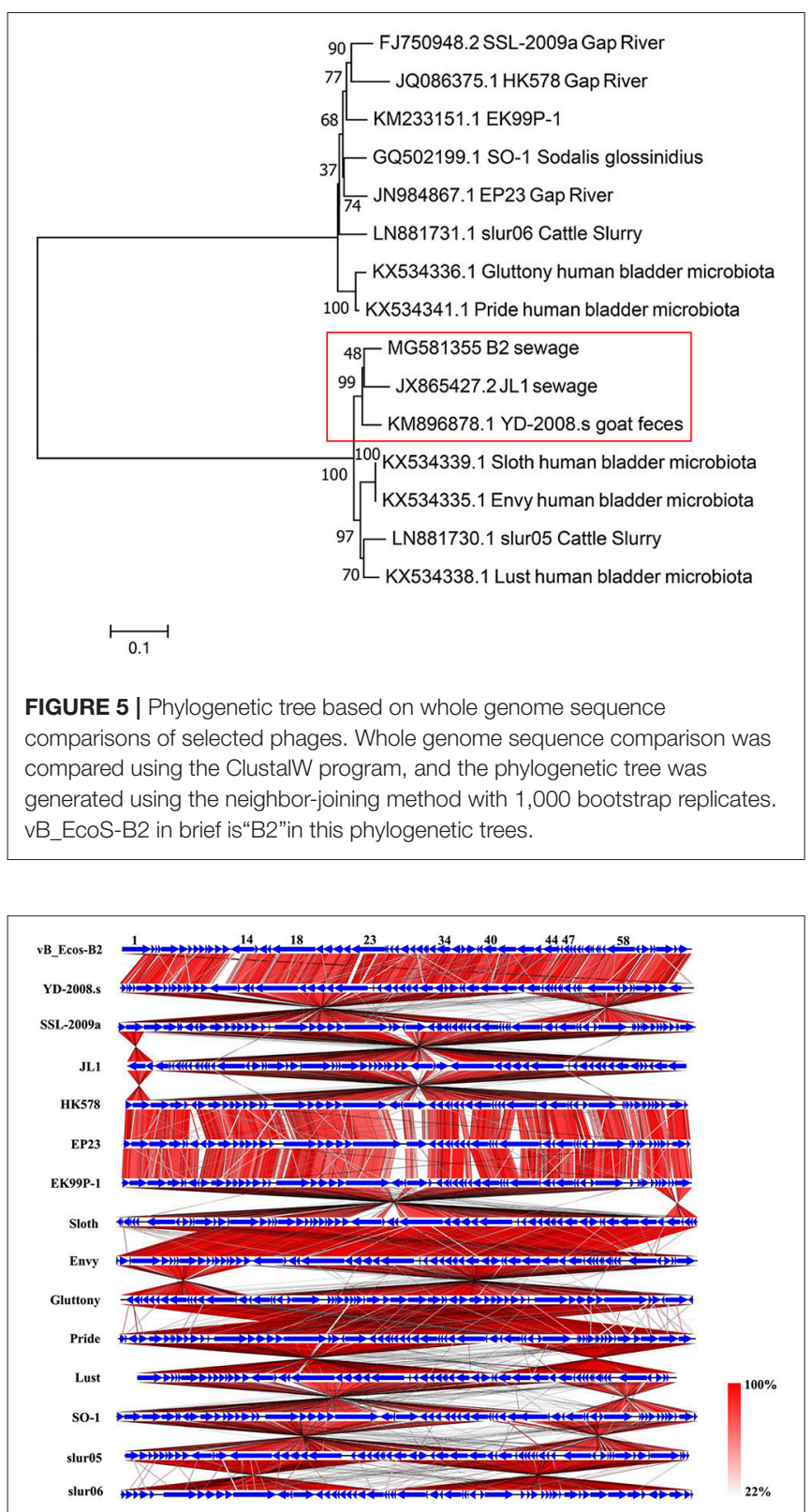

FIGURE 6 | Schematic genomic alignment of the phage vB_EcoS-B2 with other 14 homologous phages. Homologous ORFs or genes are present in blue, and the percentages of amino acid identities are shown with different colors.

host range of phage $\mathrm{vB} \_\mathrm{EcoS}-\mathrm{B} 2$ different from other relative phages.

Multiple alignment of the $\mathrm{vB} \_$EcoS-B2 and 14 relative phages showed that most of regions are highly homologous at protein levels, but they exhibit different gene arrangement with each other (Figure 6). For example, DNA polymerase I and DNA helicase regions of $\mathrm{vB} \_$EcoS-B2 were reversely matched with phage SSL-2009a. The tail protein region of phage vB_EcoS-B2 was forwardly matched with the same regions of Enterobacteria phage SSL-2009a. But head morphogenesis protein, terminase large subunit, and lysozyme regions, were back to reversely matched with phage SSL-2009a (Figure 6). Pan et al. found that the gene arrangement and genome structure of phage JL1 are different from those of the other four phages, such as SSL-2009a (Pan et al., 2013). EP23 and SSL-2009a had high similarities in amino acid sequences (95.5\% on average). But their gene orders were not conserved with each other (Chang and Kim, 2011). A lot of gene inversions and rearrangement were observed between these phages, such as YD-2008s and SSL2009a, JL1 and HK578, EK99P-1 and sloth, Envy and Gluttony, Pride and lust, SO-1and slur05 (Figure 6). Gene recombination seems to have been occurred in high frequency in these phages (Figure 6). Our results convinced that topological rearrangement of genomes has lower barriers than changes of amino acid sequences during evolution of phage $\mathrm{vB}$ _EcoS-B2 with its phage relatives ( $\mathrm{Li}$ et al., 2010; Chang and Kim, 2011; Pan et al., 2013). Gene order has major effects on growth rate for T7 phage even when genome entry is normal, suggesting that the consequence of altered gene order probably extends to fitness measures that are not tied to a short generation time (Cecchini et al., 2013). vB_EcoS-B2 and its relatives may be co-evoluted with their host through rearrangement of their genes under different selection pressures. Tailed bacteriophages constitute the most abundant and diverse group of dsDNA viruses, in which evolution is very complicated (Iranzo et al., 2016). Several results illustrated that phages may undergo genetic exchange by horizontal gene transfer from a large shared pool, and that horizontal gene transfer between phages is a component of evolution (Håggard-Ljungquist et al., 1992; Hendrix et al., 1999; Casjens, 2008; Dekel-Bird et al., 2013; Chen et al., 2016). In our study, both horizontal exchange and vertical gene order rearrangement may affect the organization of bacteriophage genomes and blur phylogenetic reconstructions in vB_EcoS-B2 and its relative phages.

In conclusion, we have isolated and characterized a new lytic phage vB_EcoS-B2 which belongs to family Siphoviridae, with lytic activity against several MDR E. coli isolates. vB_EcoSB2, like JL1 and EP23, can be assigned into virulent phages because of the presence of lysis genes such as lysozyme, putative holin-like class I protein and putative holin-like class II protein, and no similarities to lysogenic genes coding integrase, repressor, and anti-repressor proteins. The genome sequence analysis of $\mathrm{vB}$ _EcoS-B2 provided no evidence of genes related to potential virulence factors or antibiotic resistance genes. In addition, the identification of the 21 structural proteins confirmed that the vB_EcoS-B2 is a new virulent bacteriophage of $E$. coli. It also demonstrated a high degree of identity of vB_EcoS-B2 with ORFs from some other phages. Genome and proteome analysis confirmed the lytic nature of the vB_EcoS-B2. Comparative genome analysis sheds light on the mechanisms of evolutionary changes of these phage genomes. Our results indicate that gene arrangement and genome structure of phage vB_EcoS-B2 is different from that of its phage relatives. The vB_EcoS-B2 genome encodes several putative proteins, including enzymes with antimicrobial activity for the biocontrol of pathogenic bacteria or involved in the phage infection process. The phage 
genome sequence data also provide useful basic information for further research on the interaction between phages and their hosts.

\section{AUTHOR CONTRIBUTIONS}

YX, XY, YG, and XL conceived, designed and coordinated the study. YX, XY, and YG carried out the experimentation. YX, XY, YG, and XL analyzed the results. GL, XH, YX, XY, YG, and XL contributed reagents, materials, analysis tools. All authors wrote, read, and approved the final manuscript.

\section{REFERENCES}

Aggarwal, G., and Ramaswamy, R. (2002). Ab initio gene identification: prokaryote genome annotation with GeneScan and GLIMMER. J. Biosci. 27, 7-14. doi: 10.1007/BF02703679

Amarillas, L., Rubí-Rangel, L., Chaidez, C., González-Robles, A., LightbournRojas, L., and León-Félix, J. (2017). Isolation and characterization of phiLLS, a novel phage with potential biocontrol agent against multidrug-resistant Escherichia coli. Front. Microbiol. 8:1355. doi: 10.3389/fmicb.2017.01355

Andraos, N., Tabor, S., and Richardson, C. C. (2004). The highly processive DNA polymerase of bacteriophage T5. Role of the unique $\mathrm{N}$ and $\mathrm{C}$ termini. J. Biol. Chem. 279, 50609-50618. doi: 10.1074/jbc.M408428200

Birgy, A., Doit, C., Mariani-Kurkdjian, P., Genel, N., Faye, A., Arlet, G., et al. (2011). Early detection of colonization by VIM-1-producing Klebsiella pneumoniae and NDM-1-producing Escherichia coli in two children returning to France. J. Clin. Microbiol. 49, 3085-3087. doi: 10.1128/JCM.00540-11

Bolocan, A. S., Callanan, J., Forde, A., Ross, P., and Hill, C. (2016). Phage therapy targeting Escherichia coli-a story with no end? FEMS. Microbiol. Lett. 363:fnw256. doi: 10.1093/femsle/fnw256

Bourdin, G., Navarro, A., Sarker, S. A., Pittet, A. C., Qadri, F., Sultana, S., et al. (2014). Coverage of diarrhoea-associated Escherichia coli isolates from different origins with two types of phage cocktails. Microb. Biotechnol. 7, 165-176. doi: 10.1111/1751-7915.12113

Cabal, A., García-Castillo, M., Cantón, R., Gortázar, C., Domínguez, L., and Álvarez, J. (2016). Prevalence of Escherichia coli virulence genes in patients with diarrhea and a subpopulation of healthy volunteers in Madrid, Spain. Front. Microbiol. 7:641. doi: 10.3389/fmicb.2016.00641

Carrias, A., Welch, T. J., Waldbieser, G. C., Mead, D. A., Terhune, J. S., and Liles, M. R. (2011). Comparative genomic analysis of bacteriophages specific to the channel catfish pathogen Edwardsiella ictaluri. Virol. J. 8:6. doi: 10.1186/1743-422X-8-6

Casjens, S. R. (2008). Diversity among the tailed-bacteriophages that infect the Enterobacteriaceae. Res. Microbiol. 159, 340-348. doi: 10.1016/j.resmic.2008.04.005

Castanheira, M., Mendes, R. E., and Sader, H. S. (2017). Low frequency of ceftazidime-avibactam resistance among Enterobacteriaceae isolates carrying blaKPC collected in U.S. hospitals from 2012 to 2015. Antimicrob. Agents. Chemother. 61:e02369-16. doi: 10.1128/AAC.02369-16

Cecchini, N., Schmerer, M., Molineux, I. J. Springman, R., and Bull, J. J. (2013). Evolutionarily stable attenuation by genome rearrangement in a virus. G3 3, 1389-1397. doi: 10.1534/g3.113.006403

Chang, H. W., and Kim, K. H. (2011). Comparative genomic analysis of bacteriophage EP23 infecting Shigella sonnei and Escherichia coli. J. Microbiol. 49, 927-934. doi: 10.1007/s12275-011-1577-0

Chen, M., Xu, J., Yao, H., Lu, C., and Zhang, W. (2016). Isolation, genome sequencing and functional analysis of two T7-like coliphages of avian pathogenic Escherichia coli. Gene 582, 47-58. doi: 10.1016/j.gene.2016.01.049

Dalmasso, M., Strain, R., Neve, H., Franz, C. M., Cousin, F. J., Ross, R. P., et al. (2016). Three new Escherichia coli phages from the human gut show promising potential for phage therapy. PLOS ONE 11:e0156773. doi: 10.1371/journal.pone.0156773

\section{FUNDING}

This work was supported by the National Natural Science Foundation of China (81501797) and the Natural Science Foundation of Jiangsu Province (BK20151558).

\section{SUPPLEMENTARY MATERIAL}

The Supplementary Material for this article can be found online at: https://www.frontiersin.org/articles/10.3389/fmicb. 2018.00793/full\#supplementary-material

Dekel-Bird, N. P., Avrani, S., Sabehi, G., Pekarsky, I., Marston, M. F., Kirzner, S., et al. (2013). Diversity and evolutionary relationships of T7like podoviruses infecting marine cyanobacteria. Environ. Microbiol. 15, 1476-1491. doi: 10.1111/1462-2920.12103

Dufour, N., Clermont, O., La Combe, B., Messika, J., Dion, S., Khanna, V., et al. (2016). Bacteriophage LM33 P1, a fast-acting weapon against the pandemic ST131- O25b:H4 Escherichia coli clonal complex. J. Antimicrob. Chemother. 71, 3072-3080. doi: 10.1093/jac/dkw253

Dufour, N., Debarbieux, L., Fromentin, M., and Ricard, J. D. (2015). Treatment of highly virulent extraintestinal pathogenic Escherichia coli pneumonia with bacteriophages. Crit. Care Med. 43, e190-e198. doi: 10.1097/CCM.0000000000000968

Fitzgerald-Hughes, D., Bolkvadze, D., Balarjishvili, N., Leshkasheli, L., Ryan, M., Burke, L., et al. (2014). Susceptibility of extended-spectrumbeta-lactamase-producing Escherichia coli to commercially available and laboratory-isolated bacteriophages. J. Antimicrob. Chemother. 69, 1148-1150. doi: $10.1093 / \mathrm{jac} / \mathrm{dkt} 453$

Håggard-Ljungquist, E., Halling, C., and Calendar, R. (1992). DNA sequences of the tail fiber genes of bacteriophage P2: evidence for horizontal transfer of tail fiber genes among unrelated bacteriophages. J. Bacteriol. 174, 1462-1477. doi: $10.1128 /$ jb.174.5.1462-1477.1992

Haq, I. U., Chaudhry, W. N., Akhtar, M. N., Andleeb, S., and Qadri, I. (2012). Bacteriophages and their implications on future biotechnology: a review. Virol. J. 9:9. doi: 10.1186/1743-422X-9-9

Hashemolhosseini, S., Montag, D., Krämer, L., and Henning, U. (1994). Determinants of receptor specificity of coliphages of the T4 family. A chaperone alters the host range. J. Mol. Biol. 241, 524-533. doi: 10.1006/jmbi.1994.1529

Hendrix, R. W., Smith, M. C., Burns, R. N., Ford, M. E., and Hatfull, G. F. (1999). Evolutionary relationships among diverse bacteriophages and prophages: all the world's a phage. Proc. Natl. Acad. Sci. U.S.A. 96, 2192-2127. doi: 10.1073/pnas.96.5.2192

Heo, Y. J., Lee, Y. R., Jung, H. H., Lee, J., Ko, G., and Cho, Y. H. (2009). Antibacterial efficacy of phages against Pseudomonas aeruginosa infections in mice and Drosophila melanogaster. Antimicrob. Agents. Chemother. 53, 2469-2474. doi: 10.1128/AAC.01646-08

Iranzo, J., Krupovic, M., and Koonin, E. V. (2016). The double-stranded DNA virosphere as a modular hierarchical network of gene sharing. MBio 7:e0097816. doi: 10.1128/mBio.00978-16.

Jamalludeen, N., She, Y. M., Lingohr, E. J., and Griffiths, M. (2009). Isolation and characterization of virulent bacteriophages against Escherichia coli serogroups O1, O2, and O78. Poult. Sci. 88, 1694-1702. doi: 10.3382/ps.200900033

Kutateladze, M., and Adamia, R. (2010). Bacteriophages as potential new therapeutics to replace or supplement antibiotics. Trends Biotechnol. 28, 591-595. doi: 10.1016/j.tibtech.2010.08.001

Laanto, E., Hoikkala, V., Ravantti, J., and Sundberg, L. R. (2017). Long-term genomic coevolution of host-parasite interaction in the natural environment. Nat. Commun. 8:111. doi: 10.1038/s41467-017-00158-7

Lepape, A., and Monnet, D. L. (2009). Experience of European intensive care physicians with infections due to antibiotic-resistant bacteria. Euro Surveill. 14, 1-3. doi: 10.2807/ese.14.45.19393-en 
Li, S., Liu, L., Zhu, J., Zou, L., Li, M., Cong, Y., et al. (2010). Characterization and genome sequencing of a novel coliphage isolated from engineered Escherichia coli Intervirology 53, 211-220. doi: 10.1159/000 299063

Merabishvili, M., DeVos, D., Verbeken, G., Kropinski, A. M., Vandenheuvel, D., Lavigne, R., et al. (2012). Selection and characterization of a candidate therapeutic bacteriophage that lyses the Escherichiacoli O104:H4 strain from the 2011 outbreak in Germany. PLoS ONE 7:e52709. doi: 10.1371/journal.pone.0052709

Nilsson, A. S. (2014). Phage therapy-constraints and possibilities. Ups. J. Med. Sci. 119, 192-198. doi: 10.3109/03009734.2014.902878

Pan, F., Wu, H., Liu, J., Ai, Y., Meng, X., Meng, R., et al. (2013). Complete genome sequence of Escherichia coli O157:H7 lytic phage JL1. Arch. Virol. 158, 429-432. doi: 10.1007/s00705-013-1727-2

Peña, C., Gudi ol, C., Tubau, F., Saballs, M., Pujol, M., Dominguez, M. A., et al. (2006). Risk-factors for acquisition of extended-spectrum beta-lactamaseproducing Escherichia coli among hospitalised patients. Clin. Microbiol. Infect. 12, 279-284. doi: 10.1111/j.1469-0691.2005.01358.x

Postic, B., and Finland, M. (1961). Observations on bacteriophage typing of Pseudomonas aeruginosa. J. Clin. Invest. 40, 2064-2075. doi: 10.1172/JCI 104432

Pouillot, F., Chomton, M., Blois, H., Courroux, C., Noelig, J., Bidet, P., et al. (2012). Efficacy of bacteriophage therapy in experimental sepsis and meningitis caused by a clone O25b:H4-ST131 Escherichia coli strain producing CTXM-15. Antimicrob. Agents Chemother. 56, 3568-3575. doi: 10.1128/AAC. 06330-11

Regeimbal, J. M., Jacobs, A. C., Corey, B. W., Henry, M. S., Thompson, M. G., Pavlicek, R. L., et al. (2016). Personalized therapeutic cocktail of wild environmental phages rescues mice from Acinetobacter baumannii wound infections. Antimicrob. Agents Chemother. 60, 5806-5816. doi: 10.1128/AAC. 02877-15

Schooley, R. T., Biswas, B., Gill, J. J., Hernandez-Morales, A., Lancaster, J., Lessor, L., et al. (2017). Development and use of personalized bacteriophagebased therapeutic cocktails to treat a patient with a disseminated resistant Acinetobacter baumannii infection. Antimicrob. Agents Chemother. 61:e00954-17. doi: 10.1128/AAC.00954-17

Schmerer, M., Molineux, I. J., Bull, and J. J.. (2014). Synergy as a rationale for phage therapy using phage cocktails. PeerJ 2:e590. doi: 10.7717/peerj.590
Shevchenko, A., Wilm, M., Vorm, O., and Mann, M. (1996). Mass spectrometric sequencing of proteins from silver-stained polyacrylamide gels. Anal. Chem. 68, 850-858. doi: 10.1021/ac950914h

Snyder, A. B., Perry, J. J., and Yousef, A. E. (2016). Developing and optimizing bacteriophage treatment to control enterohemorrhagic Escherichia coli on fresh produce. Int. J. Food Microbiol. 236, 90-97. doi: 10.1016/j.ijfoodmicro.2016.07.023

Srinivasiah, S., Bhavsar, J., Thapar, K., Liles, M., Schoenfeld, T., and Wommac, K. E. (2008). Phages across the biosphere: contrasts of viruses in soil and aquatic environments. Res. Microbiol. 159, 349-357. doi: 10.1016/j.resmic.2008.04.010

Sullivan, M. J., Petty, N. K., and Beatson, S. A. (2011). Easyfig: a genome comparison visualizer. Bioinformatics27, 1009-1010. doi: 10.1093/bioinformatics/btr039

Sybesma, W., Zbinden, R., Chanishvili, N., Kutateladze, M., Chkhotua, A., Ujmajuridze, A., et al. (2016). Bacteriophages as potential treatment for urinary tract infections. Front. Microbiol. 7:465. doi: 10.3389/fmicb.2016.00465

Williamson, K. E., Fuhrmann, J. J., Wommack, K. E., and Radosevich, M. (2017). Viruses in soil ecosystems: an unknown quantity within an unexplored territory. Annu. Rev. Virol. 4, 201-219. doi: 10.1146/annurev-virology-101416-041639

Yang, M., Du, C., Gong, P., Xia, F., Sun, C., Feng, X., et al. (2015). Therapeutic effect of the YH6 phage in a murine hemorrhagic pneumonia model. Res. Microbiol. 166, 633-643. doi: 10.1016/j.resmic.2015.07.008

Yosef, I., Goren, M. G., Globus, R., Molshanski-Mor, S., and Qimron, U. (2017). Extending the host range of bacteriophage particles for DNA transduction. Mol. Cell 66, 721-728. doi: 10.1016/j.molcel.2017.04.025

Conflict of Interest Statement: The authors declare that the research was conducted in the absence of any commercial or financial relationships that could be construed as a potential conflict of interest.

Copyright (c) $2018 \mathrm{Xu}, \mathrm{Yu}, \mathrm{Gu}, \mathrm{Huang}, \mathrm{Liu}$ and Liu. This is an open-access article distributed under the terms of the Creative Commons Attribution License (CC $B Y)$. The use, distribution or reproduction in other forums is permitted, provided the original author(s) and the copyright owner are credited and that the original publication in this journal is cited, in accordance with accepted academic practice. No use, distribution or reproduction is permitted which does not comply with these terms. 